\title{
Measures of Health and Function that Predict Future Falls
}

\section{Matthew O’Connell, Rose Anne Kenny and Orna Donoghue}

\section{Contents}

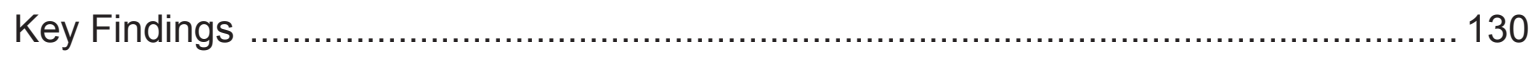

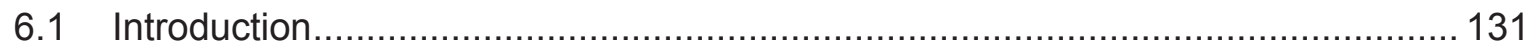

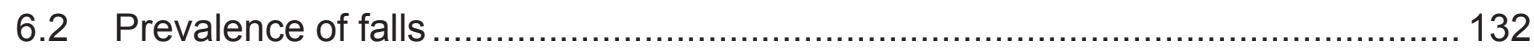

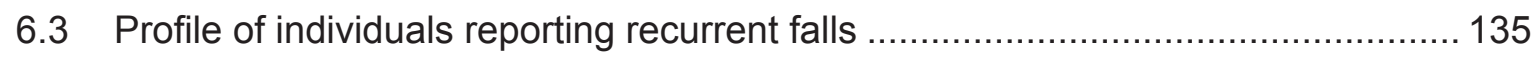

6.3.1 Co-morbidities and medications ........................................................... 135

6.3.2 Physical health and function.......................................................... 136

6.3.3 Cognitive and mental health and function ........................................ 137

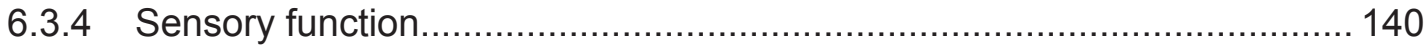

6.4 Identifying the most important predictors of recurrent falls................................ 144

6.5 Identifying the most important predictors of injurious falls.................................. 145

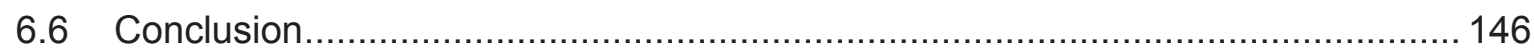

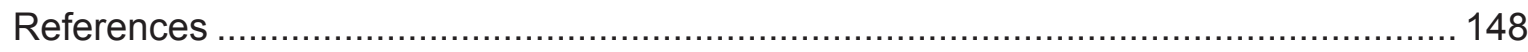




\section{Measures of Health and Function that Predict Future Falls}

\section{Key Findings}

- Falls are associated with many negative outcomes such as injury, disability, hospitalisation and reduced quality of life, therefore they are a major burden for older adults and a major challenge for the healthcare services.

- Falls are common in community-dwelling adults aged 50 years and over in Ireland, with almost 2 in 5 reporting a fall during 4 years of follow-up and 1 in 5 reporting recurrent falls and injurious falls.

- The prevalence of falls is higher in women than men and increases with age in both. Two out of five women aged 75 years and over reported recurrent falls during 4 years of follow-up while a similar number sustained a fall-related injury requiring medical attention.

- Older adults who report recurrent falls or injurious falls display poorer indicators of physical, cognitive and mental health and function compared to non-fallers.

- Many of the most important risk factors, e.g. unsteadiness when walking, depressive symptoms, non-cardiovascular conditions, fear of falling and having orthostatic hypotension, are modifiable and can be treated and improved.

- Falls assessments should be routinely conducted in older adults to identify risk factors and causes of falls so that appropriate management and fall prevention strategies can be implemented. 


\subsection{Introduction}

Falls are one of the biggest problems which occur in advancing years, because of their high frequency and adverse health consequences. Older adults are particularly vulnerable to both falls and the associated negative consequences of falls as they display age-related physiological changes such as poorer balance and slower reaction times. In addition they are more susceptible to injury due to an increased prevalence of clinical conditions such as osteoporosis, and they recover less well after injury, therefore placing them in a deconditioned state for longer (1). Falls can lead to fear of falling and activity restriction, disability, hospitalisation and reduced quality of life. An Economic Burden of Illness Study estimated that fall-related injuries in older people cost $€ 402$ million to the Irish economy in 2006 and would increase to $€ 922-1077$ million by 2020 in the absence of a National Fall and Fracture Prevention Strategy being implemented (2). This is a conservative estimate which does not include the wider economic impact incurred by carers and the longer term consequences outwith injury such as subsequent home care, medications and institutionalisation costs.

Many accidental falls occur partly due to environmental factors e.g. tripping over an uneven pavement; however, intrinsic factors relating to the individual also play a role. Previous research has identified a large number of risk factors for falls including prior history of falls; chronic conditions such as arthritis or urinary incontinence; deficits in gait or balance; reduced muscle strength; poor vision; depressive symptoms; dizziness; taking four or more medications and impaired cognition $(1,3)$. Most falls are not due to just one risk factor; instead many interact with each other and the risk of falling increases with an increasing number of risk factors. However, the most important risks appear to be impairments in gait, balance and muscle strength (1).

Despite these previous studies, there remains a lack of information on both the impact of falls and the most important risk factors at the population level in Ireland. Through its extensive survey data collection and state-of-the art health assessment, TILDA collects some of the most comprehensive information on falls risk factors available internationally.

In this chapter, the aim is to describe the frequency of falls in community-dwelling older adults in Ireland over the first three waves of data collection and subsequently, to establish a profile of fallers in relation to both self-reported and objective measures of health and function obtained at baseline (Wave 1). These measures can be roughly divided into chronic conditions and medications, physical health and function, sensory function, cognitive and mental health. We then identify the factors that are the most important 
predictors of future falls and examine if these risk factors are modifiable in an effort to best inform policy makers and clinical practice in Ireland.

In this analysis, information is obtained from both the interview and health centre assessment components of TILDA, therefore only those who participated in both components at baseline were eligible for this analysis $(n=5,035)$. Of these participants, 4,700 participated in Wave 2 (including 12 proxy interviews) and 4,400 participated in Wave 3 (including 32 proxy interviews). Proxy interviews were completed by a family member/close friend if the participant was unable to participate themselves due to physical or cognitive reasons. Participants were divided into three age groups (50-64 years, 65-74 years and 75 years or over) which reflect their ages at Wave 1.

\subsection{Prevalence of falls}

During each interview, participants were asked if they had fallen in the past year and/or since their last interview and if they had required medical attention as a result of any of these falls. At Wave 1, this information reflects an individual's history of falls in the past year. Falls reported at Waves 2 and 3 were used to define the various falls outcomes (any falls, recurrent falls, injurious falls) occurring after this baseline measurement (Table 6.1).

Table 6.1: Definition of falls outcomes

\begin{tabular}{|l|l|}
\hline Outcome & Definition \\
\hline Any Fall & $\begin{array}{l}\text { Any fall reported in the last year OR since the participant's last } \\
\text { interview. }\end{array}$ \\
\hline Recurrent Falls & $\begin{array}{l}\text { Two or more falls reported at Wave } 2 \text { or Wave } 3 \text { or both OR a } \\
\text { single fall reported at both Wave } 2 \text { and Wave } 3 .\end{array}$ \\
\hline Injurious Falls & $\begin{array}{l}\text { Any fall in which an injury serious enough to require medical } \\
\text { attention was sustained at Wave } 2 \text { or Wave } 3 .\end{array}$ \\
\hline
\end{tabular}

Table 6.2 shows the prevalence of falls, recurrent falls and injurious falls at Waves 1, 2 and 3.

Table 6.2: Prevalence of recurrent falls and injurious falls at Waves 1, 2 and 3

\begin{tabular}{|l|c|c|c|c|}
\hline & Wave 1 & Wave 2 & Wave 3 & Wave 2 and 3 \\
\hline $\mathbf{N}$ & 4,788 & 4,696 & 4,398 & 4,789 \\
\hline Any fall (\%) & 21.1 & 23.9 & 25.8 & 37.3 \\
\hline Recurrent falls (\%) & 7.7 & 9.9 & 11.1 & 19.1 \\
\hline Injurious falls (\%) & 7.7 & 10.2 & 12.1 & 18.4 \\
\hline
\end{tabular}


At Wave 1, 21\% of participants reported any fall in the year prior to interview while this figure increased to $24 \%$ at Wave 2 and $26 \%$ at Wave 3 . When considering falls which occurred at Waves 2 and 3, over one third (37\%) of participants experienced a fall during this follow-up period. At Wave $1,8 \%$ of participants reported recurrent falls in the past year and this increased to $10-11 \%$ at Waves 2 and 3 . Over the four years of follow-up (i.e. Waves 2 and 3), 19\% of participants experienced recurrent falls. The prevalence of injurious falls was very similar to recurrent falls at all waves. For the remainder of this chapter, we present the combined figure for falls occurring during follow-up i.e. based on falls occurring at Wave 2 and Wave 3.

Women were more likely to report any falls during 4 years follow-up compared to men ( $42 \%$ versus $32 \%$ ). The prevalence increased with age in both genders from $29 \%$ and $34 \%$ (in men and women aged $50-64$ years) to $42 \%$ and $60 \%$ (in men and women aged 75 years and older) (Figure 6.1). Approximately one in five older adults in Ireland (17\% of men; $21 \%$ of women) experienced recurrent falls in the last four years. Recurrent falls were more common in women aged 65-74 years and 75 years and older compared to women aged 50-64 years (Figure 6.2), however this relationship with age was weaker in men. Injurious falls were also more common in women (23\%) compared to men (13\%) but increased with age in both genders (Figure 6.3). For example, 18\% of women aged 50-64 years reported an injurious fall compared to $38 \%$ of those aged 75 years and over.

Figure 6.1: Prevalence of any fall at Wave 2 and Wave 3, by age group and sex $80.0-$ Male Female $60.0-$
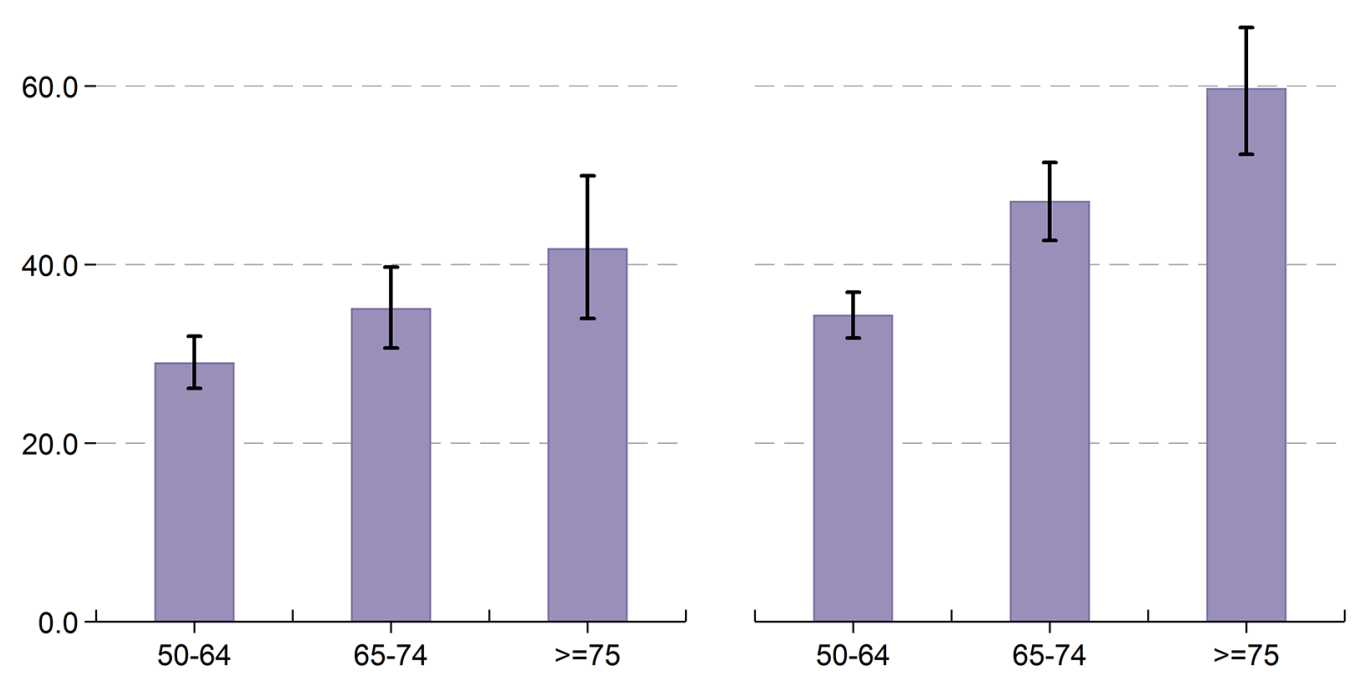

Note. $N=4789$; Missing obs = 1; Error bars correspond to $95 \%$ confidence intervals 
Figure 6.2: Prevalence of recurrent falls at Wave 2 and Wave 3, by age group and sex
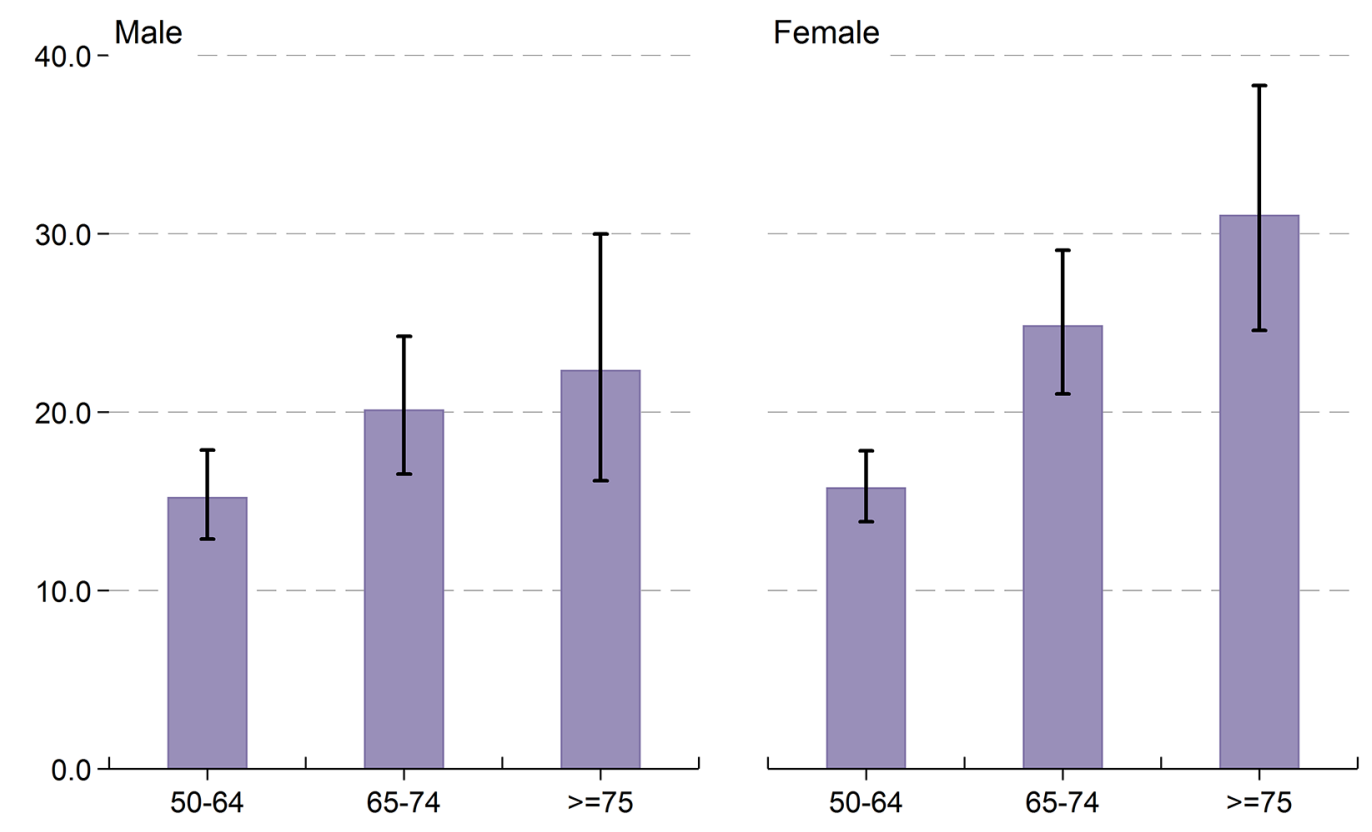

Note. $\mathrm{N}=4788$; Missing obs $=2$; Error bars correspond to $95 \%$ confidence intervals

Figure 6.3: Prevalence of injurious falls at Wave 2 and Wave 3, by age group and sex

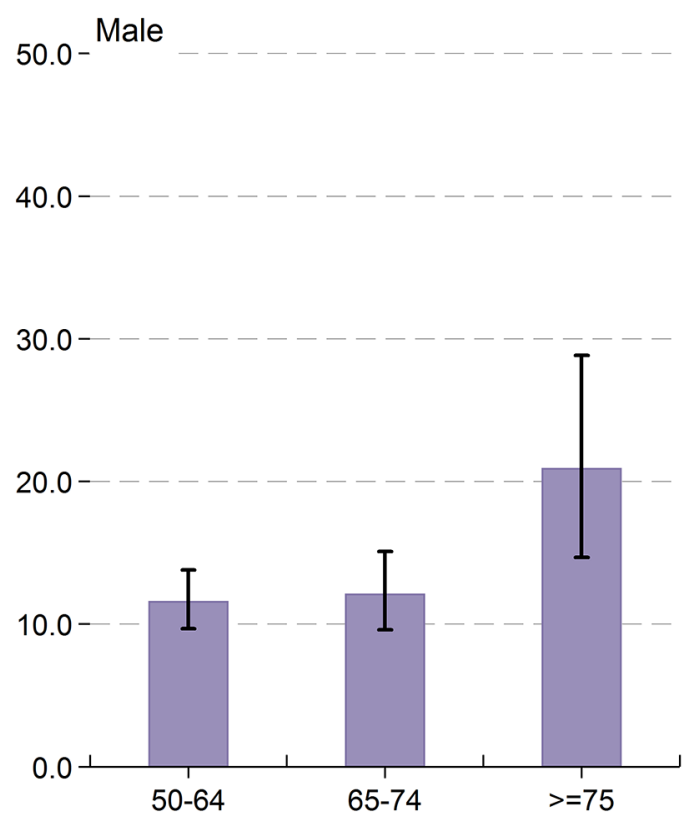

Female

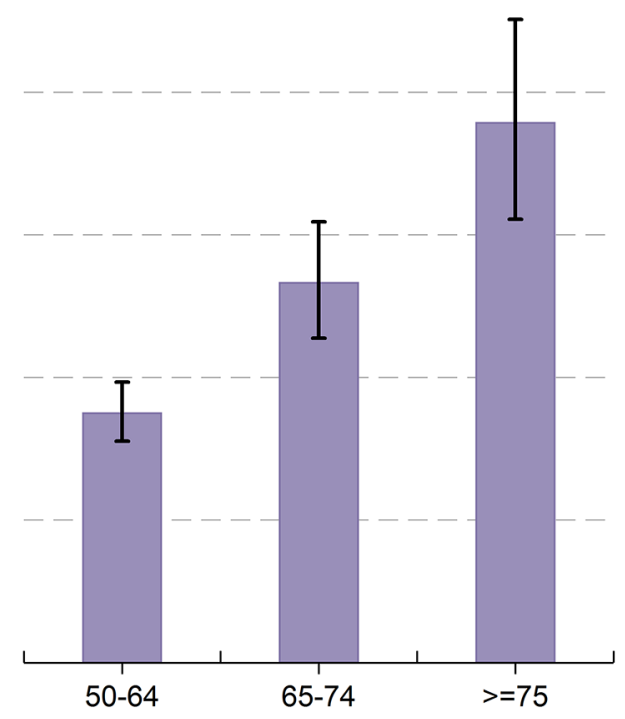

Note. $\mathrm{N}=4789$; Missing obs $=1$; Error bars correspond to $95 \%$ confidence intervals 
As shown above, falls can be defined in a number of ways. However, falls are often subject to recall bias leading to inaccuracies in reporting. In addition, a single fall can often result from an isolated event rather than a number of underlying causes. Consequently, we have chosen to focus on the more serious falls outcomes of recurrent falls and injurious falls for the remainder of this chapter.

\subsection{Profile of individuals reporting recurrent falls}

Self-reported measures were typically obtained during the interview while objective measures were obtained during the health assessment which took place in health centres in Dublin and Cork. This health assessment included tests of cardiovascular function, cognitive function, mobility, vision, bone health and anthropometry.

\subsubsection{Co-morbidities and medications}

Participants were asked to indicate if a doctor had ever told them that they had any of the following cardiovascular conditions (high blood pressure, angina, heart attack, heart failure, diabetes, stroke, mini-stroke, high cholesterol, heart murmur and heart rhythm) and non-cardiovascular conditions (chronic lung disease, asthma, arthritis, osteoporosis, cancer, Parkinson's disease). The number of conditions in each category was obtained. Participants also listed all medications taken regularly with polypharmacy defined as five or more medications.

Having a high burden of cardiovascular or non-cardiovascular conditions was strongly associated with recurrent falling. Approximately twice as many recurrent fallers had 3 or more cardiovascular conditions or 2 or more non-cardiovascular conditions, compared to non-recurrent fallers (Table 6.3). Similarly, polypharmacy was almost twice as common among recurrent fallers. This difference was most pronounced in women aged 50-64 years ( $10 \%$ in non-recurrent fallers versus $25 \%$ in recurrent fallers).

Table 6.3: Co-morbidities and medications in non-recurrent fallers and recurrent fallers

\begin{tabular}{l|cc|cc|c|c|}
\hline \multirow{2}{*}{ Co-morbidities and medications } & \multicolumn{2}{|c|}{ Non-recurrent fallers } & \multicolumn{2}{|c|}{ Recurrent fallers } & \multirow{2}{*}{$\mathbf{n}$} \\
& $\%$ & $\mathbf{( 9 5 \% ~ C l )}$ & $\%$ & $\mathbf{( 9 5 \% ~ C l )}$ & \\
\hline $\mathbf{3}$ cardiovascular conditions & 9.6 & $(8.5-10.9)$ & 18.3 & $(15.3-21.9)$ & 4,788 \\
\hline $\mathbf{2}$ non-cardiovascular conditions & 9.9 & $(8.8-11.1)$ & 18.9 & $(15.7-22.5)$ & 4,788 \\
\hline Polypharmacy & 18.2 & $(16.7-19.9)$ & 32.3 & $(28.2-36.8)$ & 4,766 \\
\hline
\end{tabular}

Note: $\mathrm{Cl}=$ confidence interval 


\subsubsection{Physical health and function}

The TILDA health assessment includes a number of high quality and novel assessments of physical health and function. Walking speed was measured using a computerised mat (active area $4.88 \mathrm{~m}$ ) with embedded pressure sensors (GAITRite ${ }^{\circledR}$, CIR Systems Inc, New York, USA). Participants started and stopped $2.5 \mathrm{~m}$ before and $2 \mathrm{~m}$ after the mat; average walking speed was obtained across two trials. Grip strength is a proxy for overall body strength and was measured using a handheld Baseline dynamometer. The average of two measurements was obtained for the dominant hand.

One of the most innovative tests included in TILDA is continuous blood pressure monitoring during active standing. A Finometer device was used to measure changes in blood pressure while standing up from a lying position. Orthostatic Hypotension $(\mathrm{OH})$ is defined as low blood pressure throughout the second minute of standing (4). Participants also underwent a quantitative heel bone ultrasound (Achilles Heel Ultrasound, Lunar, Madison, USA) to assess bone health and the risk of osteoporotic fracture. This device provides an index of bone stiffness and while this is not diagnostic, an individual is considered to have osteoporosis, osteopenia or normal bone density if the stiffness index is $<65 \%, 65-86 \%$, or $>86 \%$ respectively (5).

Height and weight were measured using standard equipment and used to calculate Body Mass Index (BMI). Physical activity was measured using the short form International Physical Activity Questionnaire (IPAQ), in which the number of days and hours spent walking and doing vigorous and moderate intensity activities in the last week was recorded (6). Specific criteria were used to classify participants into High, Moderate or Low Activity levels (7).

Recurrent fallers in the older adult population had slower walking speed and lower grip strength compared to non-recurrent fallers (Table 6.4). Orthostatic hypotension was almost twice as common among recurrent fallers, while low physical activity was slightly more common. Bone health was similar in recurrent fallers and non-recurrent fallers. Levels of obesity were also similar except when looking at adults aged 50-64 years where recurrent fallers were more likely to be obese (39\%) compared to non-recurrent fallers (33\%). Unsurprisingly, a history of falling is strongly related to future falls as $40 \%$ of recurrent fallers at Waves 2 and 3 reported a fall in the year prior to their Wave 1 interview compared to $17 \%$ of non-recurrent fallers. 
Table 6.4: Physical health and function measures in non-recurrent fallers and recurrent fallers

\begin{tabular}{|c|c|c|c|c|c|}
\hline \multirow[t]{2}{*}{ Physical health measures } & \multicolumn{2}{|c|}{ Non-recurrent fallers } & \multicolumn{2}{|c|}{ Recurrent fallers } & $\mathbf{n}$ \\
\hline & \multicolumn{4}{|c|}{ Mean $(95 \% \mathrm{Cl})$} & \\
\hline Walking speed (cm/s) & 134.4 & $(133.5-135.3)$ & 123.8 & $(121.3-126.2)$ & 4,714 \\
\hline \multirow[t]{2}{*}{ Grip strength (kg) } & 26.5 & $(26.1-26.8)$ & 23.7 & $(22.8-24.5)$ & 4,732 \\
\hline & \multicolumn{4}{|c|}{$\%(95 \% \mathrm{Cl})$} & \\
\hline Orthostatic Hypotension & 5.5 & $(4.7-6.5)$ & 9.6 & $(7.0-13.2)$ & 4,255 \\
\hline \multicolumn{6}{|l|}{ Bone health } \\
\hline Normal & 50.6 & $(48.7-52.4)$ & 46.1 & $(42.0-50.2)$ & \\
\hline Osteopenia & 40.3 & $(38.6-42.2)$ & 41.9 & $(37.6-46.3)$ & 4,760 \\
\hline Osteoporosis & 9.1 & $(7.9-10.4)$ & 12.0 & $(9.3-15.5)$ & \\
\hline \multicolumn{6}{|l|}{ Body Mass Index } \\
\hline Underweight & 0.5 & $(0.3-0.8)$ & 0.5 & $(0.2-1.3)$ & \\
\hline Normal & 22.0 & $(20.5-23.6)$ & 25.4 & $(21.8-29.4)$ & 4,780 \\
\hline Overweight & 44.5 & $(42.7-46.3)$ & 38.6 & $(34.4-42.9)$ & \\
\hline Obese & 33.1 & $(31.4-34.8)$ & 35.5 & $(31.7-39.4)$ & \\
\hline \multicolumn{6}{|l|}{ Physical activity level } \\
\hline Low & 28.4 & $(26.6-30.4)$ & 34.7 & $(30.7-39.0)$ & \\
\hline Moderate & 35.8 & $(34.0-37.7)$ & 36.4 & $(32.6-40.3)$ & 4,748 \\
\hline High & 35.7 & $(33.6-37.5)$ & 28.9 & $(25.3-32.8)$ & \\
\hline Fall in 12 months prior to Wave 1 & 16.5 & $(15.1-18.0)$ & 40.3 & $(36.2-44.6)$ & 4,786 \\
\hline
\end{tabular}

\subsubsection{Cognitive and mental health and function}

Global cognition was assessed using the Montreal Cognitive Assessment (MoCA) which assesses ability across multiple domains of cognitive function: memory recall, visuospatial ability, executive function, attention, language, and orientation to time/place. Executive function allows an individual to plan, organize and complete a task. It is often associated with balance and walking-based tasks and therefore poor executive function is a risk factor for falls. It was assessed using the Colour Trails Test which has two components, both of which involve connecting a series of numbered and coloured circles. The difference between the two components (Colour Trails time difference) reflects executive function (8). 
Concentration and processing speed were assessed using a computer-based choice reaction time test. Participants pressed a button on the keyboard until an onscreen stimulus appeared (YES/NO); then they released the button and pressed the corresponding YES/NO button on the keyboard. Total response time is the time taken from when the stimulus appeared, for the participant to release the button and press the target button on the keyboard.

Recurrent fallers in the older adult population had lower scores on MoCA (lower global cognitive function) and a larger Colour Trails time difference (poorer executive function) (Table 6.5). There were no gender differences in global cognition, executive function or reaction time, however there was a clear association between increasing age and poorer function in all three cognitive tests in both recurrent fallers and non-recurrent fallers. The relationship between age and Colour Trail time difference is shown in Figure 6.4.

Table 6.5: Cognitive function in non-recurrent fallers and recurrent fallers

\begin{tabular}{|c|c|c|c|c|c|}
\hline \multirow{2}{*}{ Cognitive function } & \multicolumn{2}{|c|}{ Non-recurrent fallers } & \multicolumn{2}{|c|}{ Recurrent fallers } & \multirow{2}{*}{$\mathbf{n}$} \\
\hline & $\%$ & $(95 \% \mathrm{CI})$ & & $(95 \% \mathrm{Cl})$ & \\
\hline MoCA (max score 30 ) & 24.7 & $(24.6-24.9)$ & 24.1 & $(23.7-24.4)$ & 4,772 \\
\hline Colour trail time difference (s) & 56.3 & $(55.1-57.6)$ & 61.3 & $(58.2-64.4)$ & 4,723 \\
\hline Choice reaction time (ms) & 844.2 & (829.0-859.5) & 891.6 & $(859.0-924.1)$ & 4,694 \\
\hline
\end{tabular}

Note: $\mathrm{Cl}=$ confidence interval 
Figure 6.4: Executive function (indicated by colour trails time difference) in non-recurrent fallers and recurrent fallers, by age group
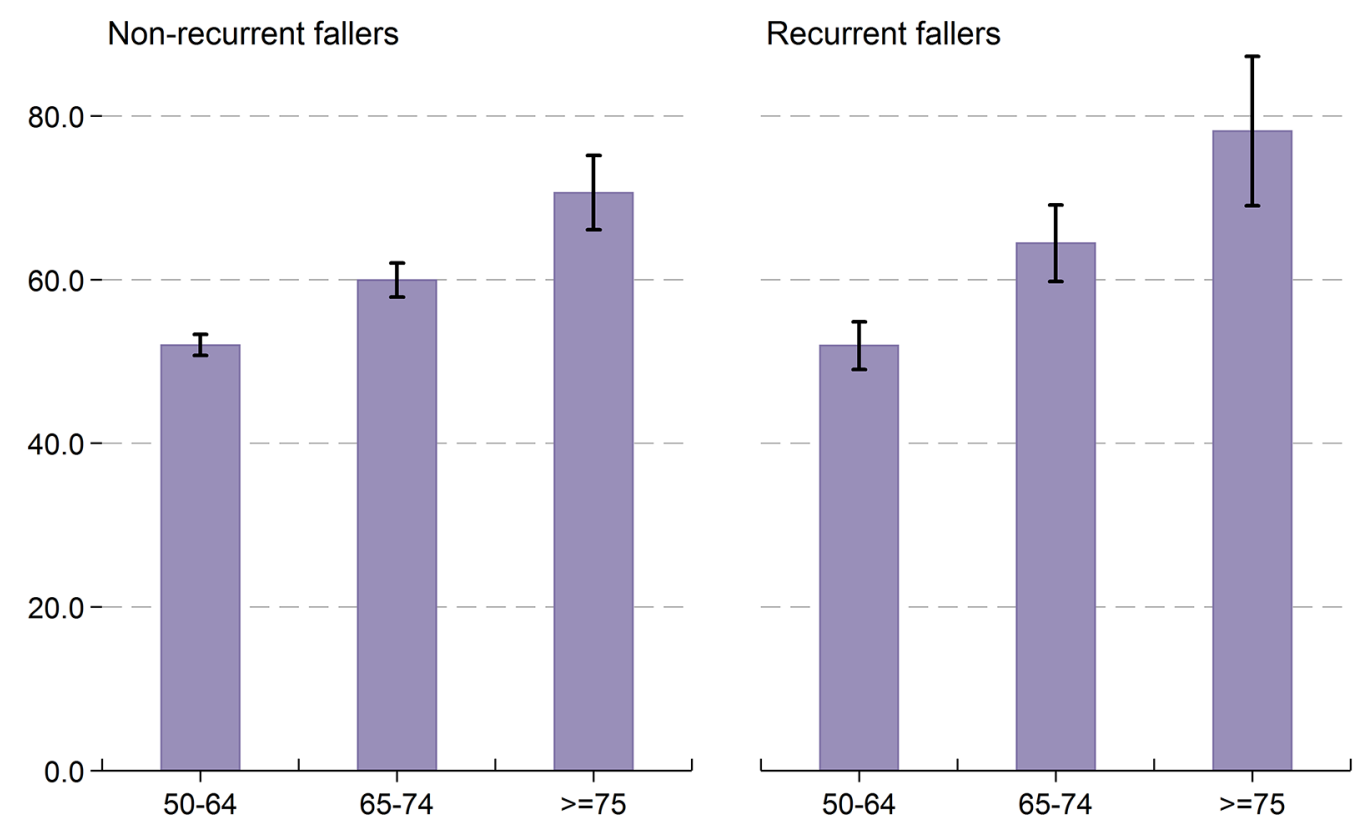

Note. $N=4723$; Missing obs $=67$; Error bars correspond to $95 \%$ confidence intervals

Depressive symptoms were assessed using the 20-item Centre for Epidemiological Studies Depression (CES-D) scale, where the maximum score is 60 (9). Participants scoring $\geq 16$ were classified as having moderate or potentially clinically relevant depressive symptoms while those scoring 8-15 were classified as having mild or sub-threshold depressive symptoms. Fear of falling was identified by asking "Are you afraid of falling?" and if so, to what extent (somewhat afraid or very much afraid).

Participants who reported recurrent falls at follow-up were more likely to report potentially clinically relevant depressive symptoms and to be somewhat or very much afraid of falling compared to non-recurrent fallers (Table 6.6). Depressive symptoms did not vary by age group or sex in recurrent fallers, however being somewhat afraid of falling was reported more frequently in women (36\% versus $18 \%$ in men) and in the older age categories $(41 \%$ in fallers aged $\geq 75$ years versus $20 \%$ in fallers aged $50-64$ years). 
Table 6.6: Depressive symptoms and fear of falling in non-recurrent fallers and recurrent fallers

\begin{tabular}{|c|c|c|c|c|c|}
\hline \multirow{2}{*}{ Mental health } & \multicolumn{2}{|c|}{ Non-recurrent fallers } & \multicolumn{2}{|c|}{ Recurrent fallers } & \multirow{2}{*}{$\mathbf{n}$} \\
\hline & $\%$ & $(95 \% \mathrm{Cl})$ & $\%$ & $(95 \% \mathrm{Cl})$ & \\
\hline \multicolumn{6}{|c|}{ Depressive symptoms (\%) } \\
\hline None & 73.4 & $(71.6-75.2)$ & 62.8 & $(58.5-66.8)$ & \\
\hline Mild (sub-threshold) & 16.9 & $(15.5-18.4)$ & 19.3 & $(16.0-23.1)$ & 4,788 \\
\hline $\begin{array}{l}\text { Moderate (potentially } \\
\text { clinically relevant) }\end{array}$ & 9.7 & $(8.5-11.0)$ & 17.9 & $(14.8-21.5)$ & \\
\hline \multicolumn{6}{|l|}{ Fear of falling (\%) } \\
\hline None & 81.5 & $(79.6-83.3)$ & 63.2 & $(58.5-67.7)$ & \\
\hline Somewhat afraid & 14.8 & $(13.3-16.6)$ & 28.4 & $(24.0-33.2)$ & 4,787 \\
\hline Very much afraid & 3.6 & $(2.8-4.8)$ & 8.3 & $(6.1-11.3)$ & \\
\hline
\end{tabular}

Note: $\mathrm{Cl}=$ confidence interval

\subsubsection{Sensory function}

During the interview, participants rated their steadiness when walking (10) with unsteadiness indicated if participants reported being Slightly steady, Slightly unsteady or Very unsteady (as opposed to Very steady). Participants also self-rated their vision and hearing with answers dichotomised into two categories: Excellent/Very Good/Good and Fair/Poor. One participant who reported being legally blind was included in the Fair/Poor vision category.

Two measures of visual function were assessed during the health assessment. Visual acuity, which is the acuteness or clearness of vision, was assessed using a LogMAR (Minimal Angle of Resolution) chart which is often used by optometrists and GPs.

Participants stood 4 metres away from the chart and completed the test with each eye, using corrective glasses/lenses if required. The logMAR score for the best eye was converted into a Visual Analog Score (VAS) where higher scores represent better visual acuity (11). Contrast sensitivity is the ability to distinguish an object from the background in low contrast conditions e.g. at dusk. It was measured using a Functional Vision Analyser (Stereo Optical, Chicago, IL, USA) under mesopic $\left(3 \mathrm{~cd} / \mathrm{m}^{2}\right)$ background illumination conditions in non-glare and glare conditions. The test provides 10 scores obtained at different spatial frequencies (1.5, 3, 6, 12 and 18 cycles per degree, cpd). Sharp images with small details contain high spatial frequency information while coarser images with 
blurred details contain low spatial frequency information. We subsequently used factor analysis to identify two distinct features for each individual corresponding to their "high frequency" and "low frequency" contrast sensitivity (12). Each factor score was divided into quartiles with the lowest quartile representing poorer contrast sensitivity.

Overall, recurrent fallers in the older adult population in Ireland were more likely to report unsteadiness when walking compared to non-recurrent fallers although there was no difference in self-rated vision or hearing (Table 6.7).

Table 6.7: Sensory function in non-recurrent fallers and recurrent fallers

\begin{tabular}{|c|c|c|c|c|c|}
\hline \multirow{2}{*}{ Sensory function } & \multicolumn{2}{|c|}{ Non-recurrent fallers } & \multicolumn{2}{|c|}{ Recurrent fallers } & \multirow{2}{*}{$\mathbf{n}$} \\
\hline & $\%$ & $(95 \% \mathrm{Cl})$ & $\%$ & $(95 \% \mathrm{Cl})$ & \\
\hline Self-rated unsteadiness & 14.7 & $(13.1-16.4)$ & 37.4 & $(33.0-42.1)$ & 4,785 \\
\hline Self-rated vision (poor/fair) & 8.8 & $(7.7-10.1)$ & 12.4 & $(9.8-15.7)$ & 4,788 \\
\hline Self-rated hearing (poor/fair) & 14.5 & $(13.2-16.0)$ & 19.0 & $(15.8-22.8)$ & 4,788 \\
\hline Visual acuity score & 96.0 & $(95.7-96.4)$ & 95.1 & $(94.2-95.9)$ & 4,771 \\
\hline \multicolumn{6}{|l|}{ Contrast sensitivity (no glare) } \\
\hline $1.5 \mathrm{cpd}$ & 36.1 & $(35.4-36.9)$ & 34.2 & $(32.4-36.0)$ & \\
\hline $3 \mathrm{cpd}$ & 66.6 & $(65.4-67.8)$ & 62.4 & $(59.7-65.0)$ & \\
\hline $6 \mathrm{cpd}$ & 31.7 & $(30.8-32.7)$ & 27.6 & $(25.7-29.5)$ & 4,561 \\
\hline $12 \mathrm{cpd}$ & 6.5 & $(6.1-6.8)$ & 5.3 & $(4.4-6.3)$ & \\
\hline $18 \mathrm{cpd}$ & 0.7 & $(0.7-0.8)$ & 0.6 & $(0.4-0.7)$ & \\
\hline \multicolumn{6}{|l|}{ Contrast sensitivity (glare) } \\
\hline $1.5 \mathrm{cpd}$ & 37.9 & $(37.2-38.6)$ & 33.8 & $(32.3-35.2)$ & \\
\hline $3 \mathrm{cpd}$ & 68.9 & $(67.6-70.2)$ & 62.6 & $(59.8-65.3)$ & \\
\hline $6 \mathrm{cpd}$ & 32.1 & $(31.1-33.1)$ & 27.8 & $(25.9-29.6)$ & 4,553 \\
\hline $12 \mathrm{cpd}$ & 6.9 & $(6.5-7.3)$ & 5.1 & $(4.4-5.7)$ & \\
\hline $18 \mathrm{cpd}$ & 0.8 & $(0.7-0.9)$ & 0.5 & $(0.4-0.6)$ & \\
\hline
\end{tabular}

Note: $\mathrm{Cl}=$ confidence interval 
Older men and women who were recurrent fallers were equally as likely to report unsteadiness while walking ( $36 \%$ versus $39 \%$ ) and fair/poor vision (14\% versus $11 \%)$, however women were less likely to report fair/poor hearing than men (14\% versus $26 \%$ ). In general, unsteadiness became more prevalent with increasing age especially in recurrent fallers (Figure 6.5), although the relationship was weaker for fair/poor vision and hearing.

Figure 6.5: Self-reported unsteadiness while walking in non-recurrent fallers and recurrent fallers, by age group

$\square$ Steady Unsteady

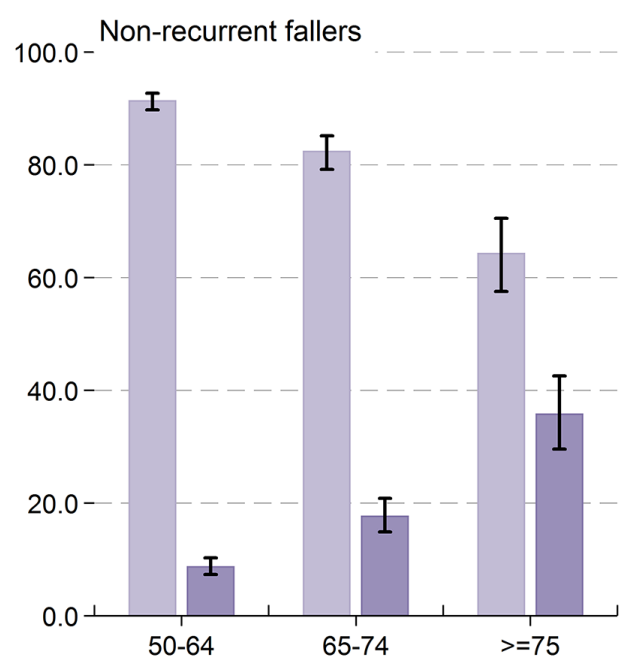

Recurrent fallers

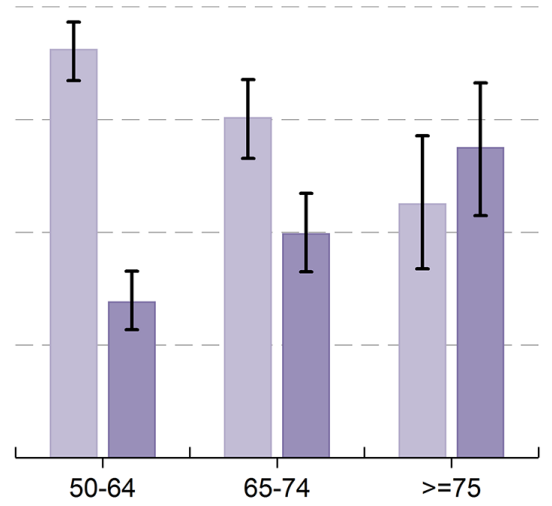

Note. $\mathrm{N}=4785$; Missing obs $=5$; Error bars correspond to $95 \%$ confidence intervals 
At most spatial frequencies of visual function assessed in TILDA (i.e. across the spectrum representing sharp detailed images to coarser images), contrast sensitivity was lower in recurrent fallers compared to non-recurrent fallers but there was no difference in visual acuity (Table 6.7). However, there were clear age and sex differences with lower visual acuity and contrast sensitivity observed with increasing age and in women (Figure 6.6).

Figure 6.6: Visual acuity in non-recurrent fallers and recurrent fallers, by age group and sex
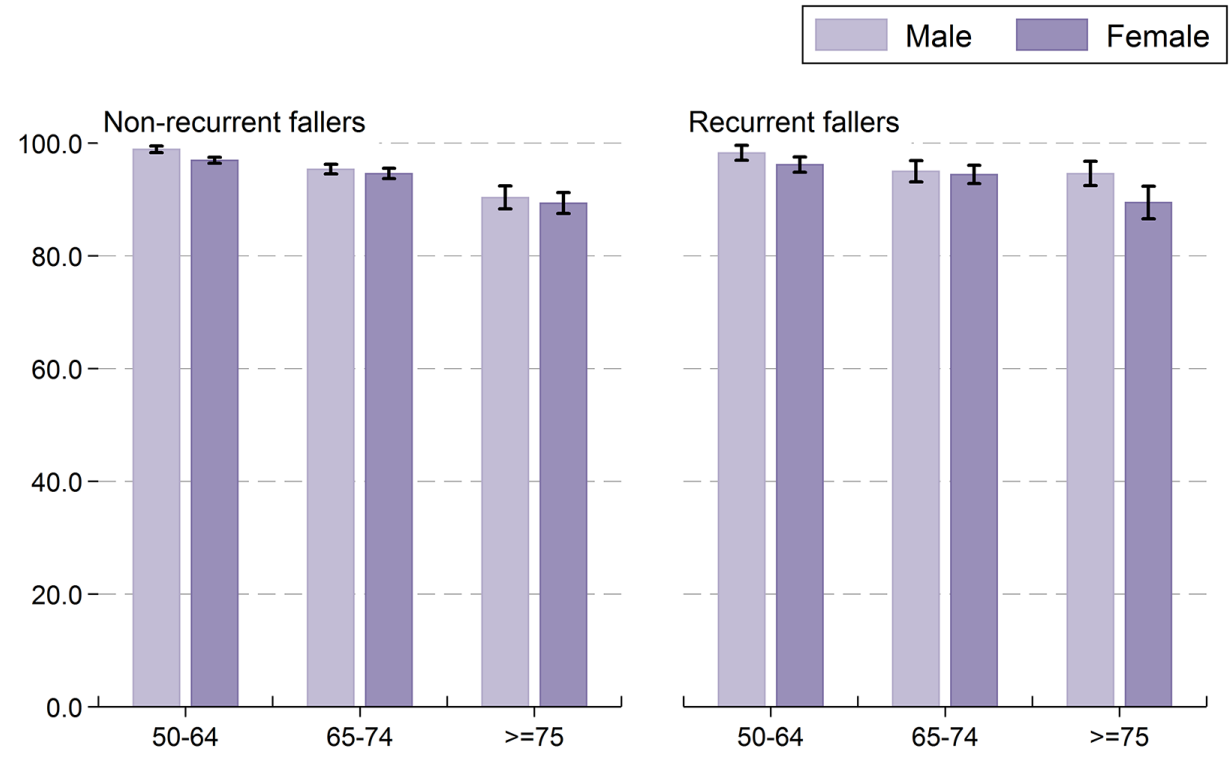

Note. $\mathrm{N}=4771$; Missing obs $=19$; Error bars correspond to $95 \%$ confidence intervals 


\subsection{Identifying the most important predictors of recurrent falls}

All of the variables used above to profile fallers were included in a multivariate model to identify the most important predictors of recurrent falls at Wave 2 and/or Wave 3 . These were identified as previous history of falling, fear of falling, self-reported unsteadiness while walking, and non-cardiovascular chronic conditions (Table 6.8). The Relative Risks $(\mathrm{RR})$ presented in the table can be interpreted as the change in risk associated with having the risk factor mentioned. For example, participants who reported unsteadiness while walking had a 1.5 times, or $50 \%$, increased risk of reporting recurrent falls compared to those who did not report unsteadiness while walking. It should be noted that this relatively simple modelling approach does not take account of potentially important modifying effects of different variables in the model, nor of potential causal pathways between variables. Future analyses from TILDA will use more sophisticated statistical methods to better understand how these risk factors may vary depending on their interactions with certain characteristics e.g. sex, age group, depressive symptoms.

Table 6.8: Indicators of health and function which predict recurrent falls at Wave 2 and/or Wave 3

\begin{tabular}{|l|c|c|}
\hline & RR & (95\% Cl) \\
\hline Self-reported unsteadiness when walking & 1.50 & $(1.22-1.85)$ \\
\hline $\mathbf{1}$ non-cardiovascular condition & 1.47 & $(1.21-1.79)$ \\
\hline $\mathbf{2}$ non-cardiovascular conditions & 1.55 & $(1.21-1.97)$ \\
\hline Somewhat afraid of falling & 1.31 & $(1.07-1.59)$ \\
\hline Very afraid of falling & 1.50 & $(1.13-1.99)$ \\
\hline Previous history of falls & 2.03 & $(1.72-2.38)$ \\
\hline
\end{tabular}

Full model includes age, sex, BMI, previous history of falls, number of cardiovascular conditions, number of non-cardiovascular conditions, orthostatic hypotension, osteopenia/osteoporosis, grip strength, usual walking speed, self-rated unsteadiness when walking, self-rated hearing, self-rated vision, visual acuity, contrast sensitivity, Montreal Cognitive Assessment, colour trails time difference, choice reaction time, depressive symptoms, fear of falling and polypharmacy $(n=3,858)$. 


\subsection{Identifying the most important predictors of injurious falls}

The profile of injurious fallers was very similar to recurrent fallers. They also displayed poorer indicators of health and function across multiple domains including physical, cognitive and mental health. We then investigated the predictors of injurious falls, again using a multivariate analysis. The most important variables that predict injurious falls at follow-up are age, being female, a previous history of falls, having orthostatic hypotension, clinically relevant depressive symptoms, osteopenia and self-reported unsteadiness when walking (Table 6.9).

Table 6.9: Indicators of health and function which predict injurious falls at Wave 2 and/or Wave 3

\begin{tabular}{|l|cc|}
\hline & RR & $(\mathbf{9 5 \%} \mathrm{CI})$ \\
\hline Orthostatic hypotension & 1.56 & $(1.22-2.00)$ \\
\hline Self-reported unsteadiness when walking & 1.32 & $(1.06-1.64)$ \\
\hline Clinically relevant depressive symptoms & 1.35 & $(1.05-1.74)$ \\
\hline Osteopenia & 1.26 & $(1.05-1.50)$ \\
\hline Age (per 1 year increase) & 1.02 & $(1.01-1.03)$ \\
\hline Female sex & 1.54 & $(1.27-1.87)$ \\
\hline Previous history of falls & 1.82 & $(1.54-2.15)$ \\
\hline
\end{tabular}

Full model includes age, sex, BMI, previous history of falls, number of cardiovascular conditions, number of non-cardiovascular conditions, orthostatic hypotension, osteopenia/osteoporosis, grip strength, usual walking speed, self-rated unsteadiness when walking, self-rated hearing, self-rated vision, visual acuity, contrast sensitivity, Montreal Cognitive Assessment, colour trails time difference, choice reaction time, depressive symptoms, fear of falling and polypharmacy $(n=3,858)$. 


\subsection{Conclusion}

The burden of falls is high in adults aged 50 years and over in Ireland, with almost $40 \%$ reporting at least one fall during 4 years follow-up. Importantly, approximately half of fallers or nearly $20 \%$ of the population reported recurrent falls, a well known risk factor for subsequent injury and hospitalisation. A similar proportion experienced a fall that resulted in an injury serious enough to require medical treatment. In general, older adults who report recurrent falls or injurious falls display poorer indicators of physical, cognitive and mental health and function compared to non-fallers. The most important factors that predict recurrent falls are a history of previous falls, self-reported unsteadiness when walking, an increasing number of non-cardiovascular conditions, and being somewhat afraid of falling. Injurious fallers share many of these risk factors (history of previous falls, self-reported unsteadiness when walking), however being older, female, having potentially clinically relevant depressive symptoms, reduced bone strength (osteopenia) and having orthostatic hypotension are also important predictors in this group.

The importance of a previous history of falls highlights the need for early monitoring and intervention to address any existing risk factors. Most falls occur while walking (13), therefore unsteadiness while walking presents an opportunity to identify an underlying difficulty or perception that something is not quite right, even if this is not visually obvious. Depressive symptoms are common in older adults and they have a complex relationship with falls. Both share common risk factors; depressive symptoms and consequent treatment with antidepressants can lead to a fall, while falls can also result in increased depressive symptoms (14). Orthostatic hypotension is also common in older adults and may cause falls and faints through decreases in brain blood flow when standing up.

Falls have very serious consequences, therefore, it is important to identify someone with a high risk of falls and subsequently implement an appropriate fall prevention programme to reduce their risk factors where possible. The current American Geriatric Society and British Geriatric Society Guidelines for Fall Prevention (15) recommend that all adults aged 65 years and over should be asked about falls in the past 12 months and difficulties with gait or balance, at least once per year. Those who have fallen previously or who report difficulties with gait or balance should then undergo a comprehensive falls assessment to identify the need for an intervention to reduce their fall risk. Ideally, this would be implemented at the primary care level to promote a proactive approach that focuses on early detection of risk rather than a reactive approach after a fall has occurred. Luckily, many of the risk factors highlighted above are modifiable and can at least be improved if not removed altogether. 
It is important to highlight that falls and physiological decline are not an inevitable consequence of ageing. The most effective fall prevention strategies are multifactorial and may include interventions to improve muscle strength, gait and balance; reduce medications (where possible); manage postural hypotension; manage foot problems and footwear; adapt or modify the home environment and supplement with Vitamin D (American Geriatric Society/British Geriatric Society Guidelines, 2011). Physical exercise is one of the most effective means of improving walking, balance and strength while also providing a range of other physical, cognitive, psychological and social benefits $(16,17$, 18). Assuming that it is adapted to each individual's needs, it should be recommended as standard for all individuals, young and old. However, Rose highlighted that the most effective type, intensity and dose of exercise is not yet clear and therefore is likely to vary with different levels of fall risk (19).

Given the changing demographics, falls represent a major challenge for the healthcare services. In 2008, the Health Service Executive, Department of Health and Children and National Council on Ageing and Older People published a Strategy to Prevent Falls and Fractures in Ireland's Ageing Population. This relies on identification of risk factors, management of specific conditions and appropriate intervention to help people to reduce their risk of falls. The National Falls and Bone Health Project AFFINITY (Activating Falls and Fracture Prevention in Ireland Together) was set up in 2013 to implement this strategy (20). However to date, fall risk assessment is not routinely carried out, therefore many older adults who would benefit from intervention and/or modification of risk factors are not being flagged for follow-up.

More recently, the National Clinical Programme for Older People which is a joint initiative between the HSE Clinical Strategy and Programmes Division and the Royal College of Physicians of Ireland, have highlighted the role of the comprehensive geriatric assessment. This reviews an older person's medical conditions, mental health, functional capacity and social circumstances with the intention of developing and implementing an integrated assessment, intervention and review of an older person's individual needs (21). This encompasses many of the same tests that are used in falls assessment and therefore, there is a lot of scope to identify fall risk using this approach.

In summary, falls and fall-related injuries are common among older adults in Ireland and there are multiple health-related factors that may increase an individual's risk of falls. Excitingly, many of the factors identified are potentially modifiable, paving the way for future interventions and falls prevention initiatives. 


\section{References}

1. Rubenstein LZ. Falls in older people: epidemiology, risk factors and strategies for prevention. Age Ageing. 2006;35 Suppl 2:ii37-ii41.

2. Health Service Executive, National Council on Ageing and Older People, Department of Health and Children. Strategy to Prevent Falls and Fractures in Ireland's Ageing Population. Report of the National Steering Group on the Prevention of Falls in Older People and the Prevention and Management of Osteoporosis throughout Life, June 2008.

3. Tinetti ME. Preventing falls in elderly persons. N Engl J Med. 2003; 348(1):42-49.

4. Finucane C, O'Connell MD, Fan CW, Savva GM, Soraghan CJ, Nolan H, Cronin H, Kenny RA. Age related Normative changes in phasic orthostatic blood pressure in a large population study: Findings from the Irish Longitudinal Study on Ageing (TILDA). Circulation. 2014;130:1780-1789

5. Varenna M, Sinigaglia L, Adami S, Giannini S, Isaia G, Maggi S, et al. Association of quantitative heel ultrasound with history of osteoporotic fractures in elderly men: the ESOPO study. Osteoporo Int. 2005;16(12):1749-1754.

6. Craig CL, Marshall AL, Sjostrom M, Bauman AE, Booth ML, Ainsworth BE, et al. International physical activity questionnaire: 12-country reliability and validity. Med Sci in Sports Exerc. 2003;35(8):1381-95.

7. IPAQ. Guidelines for the data processing and analysis of the International Physical Activity Questionnaire 2005.

8. D’Elia LF, Satz P, Uchiyama CL, White T. Color Trails Test. Professional manual. Odessa, FL: Psychological Assessment Resources, 1996.

9. Radloff LS. The CES-D scale: A self-report depression scale for research in the general population. Applied Psychological Measurement. 1977;1:385-401.

10. Clark DO, Callahan CM, Counsell SR., Reliability and validity of a steadiness score. Am Geriatr Soc. 2005;53(9):1582-6. 
11. Colenbrander A. Visual standards, aspects and range of vision loss with emphasis on population surveys. Report prepared for the International Council of Ophthalmology at the 29th International Congress of Ophthalmology. Sydney, 2002.

12. Donoghue OA, Ryan H, Duggan E, Finucane C, Savva GM, Cronin H, Loughman J, Kenny RA. Relationship between fear of falling and mobility varies with visual function among older adults. Geriatr and Gerontol Int. 2014;14(4):827-36

13. Mackenzie L, Byes J, Higginbotham N. A prospective community-based study of falls among older people in Australia: Frequency, circumstances, and consequences. OTJR: Occupation, Participation, and Health. 2002;22(4):143-152.

14. laboni A, Flint AJ. The complex interplay of depression and falls in older adults: a clinical review. Am J Geriatr Psychiatry 2013;21:484-492.

15. Summary of the Updated American Geriatrics Society/British Geriatrics Society clinical practice guideline for prevention of falls in older persons. Am Geriatr Soc. 2011;59(1), 148-157.

16. Colcombe S, Kramer AF. Fitness effects on the cognitive function of older adults: a meta-analytic study. Psychol Sci. 2003;14:125-130.

17. Donoghue O, O'Connell M, Kenny RA. Walking to Wellbeing: Physical Activity, Social Participation and Psychological Health in Irish adults aged 50 years and Older. Dublin: The Irish Longitudinal Study on Ageing, 2016.

18. World Health Organisation. Global Recommendations on Physical Activity for Health. 2010.

19. Rose DJ. Preventing falls among older adults: No "one size suits all" intervention strategy. Rehabil Res Dev. 2008;45(8):1153-1166.

20. National Falls and Bone Health Project AFFINITY (Activating Falls and Fracture Prevention in Ireland Together). Available at: http://www.affinityfallsbonehealth.ie/index. html.

21. National Clinical Programme for Older People (2016). Comprehensive Geriatric Assessment Available from: https://www.hse.ie/eng/about/Who/clinical/natclinprog/ olderpeopleprogramme/resources/ComprehensiveGeriatricAssessmentSummary.pdf 
